International Journal of Current Advanced Research

ISSN: O: 2319-6475, ISSN: P: 2319 - 6505, Impact Factor: SJIF: 5.995

Available Online at www.journalijcar.org

Volume 6; Issue 3; March 2017; Page No. 2721-2723

DOI: http://dx.doi.org/10.24327/ijcar.2017.2723.0088

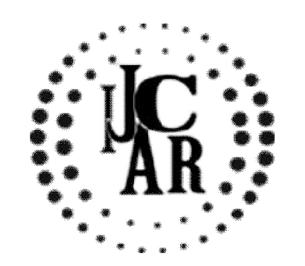

Research Article

\title{
PHENOTYPIC CHARACTERISTICS OF ENTEROCOCCUS FAECALIS ISOLATED FROM ENDODONTIC INFECTIONS
}

\author{
Harini.K and Geetha.R.V
}

Saveetha Dental College and Hospitals, Chennai, India

\begin{tabular}{l} 
A R T I C L E I N F O \\
\hline Article History: \\
Received $20^{\text {th }}$ December, 2016 \\
Received in revised form $24^{\text {th }}$ January, 2017 \\
Accepted $6^{\text {th }}$ February, 2017 \\
Published online $28^{\text {th }}$ March, 2017
\end{tabular}

\section{Key words:}

Haemolysis, Enterococcus faecalis,

Gelatinase, Endodontic infection.

\begin{abstract}
A B S T R A C T
Aim and objective: To evaluate the phenotypic characteristics of Enterococcus faecalis isolated from endodontic infections.

Background: Enterococcus faecalis is a microorganism detected in asymptomatic, persistent endodontic infections. E. faecalis has an ability to survive harsh environments including extreme alkaline pH.It resists bile salts, detergents, heavy metals, ethanol, azide $\&$ desiccation.E. faecalis endures prolonged period of nutritional deprivation. Enterococci can grow at $10 \mathrm{C}$ and $45 \mathrm{C}$ at $\mathrm{pH} 9.6$ in $6.5 \% \mathrm{NaCl}$ broth and survive at $60 \mathrm{C}$ for 30 minutes. This may explain its survival in root canal infections, where nutrients are scarce and there are limited means of escape from root canal medicaments.

Materials and methods: A total of 20 samples were collected from patients undergoing endodontic treatment. The samples were inoculated in Mac Conkey agar and brain heart infusion agar. Presumptive identification of Enterococci was done by Gram's stain, Catalase test and Heat tolerance test in which Enterococci are gram positive cocci arranged in pairs. Brain-heart infusion agar (Oxoid) supplemented with 5\% sheep blood was used for the detection of haemolytic activity. Gelatinase assay was carried out by adding an inoculum from a pure culture into tubes containing $12 \%$ gelatin in $0.8 \%$ nutrient broth.

Results: Cytolysin production was screened by cultivation on blood agar,2(10\%) isolates produced complete $(\beta)$ haemolysis, $1(5 \%)$ isolate produced partial $(\alpha)$ haemolysis and the rest of the isolates did not produce haemolysis on blood agar $(\gamma)$ haemolysis. Gelatinase assay by gelatin hydrolysis test revealed that $4(20 \%)$ of the isolates phenotypically expressed gelatinase gene as they could not liquefy gelatin media.
\end{abstract}

Copyright $₫ 2017$ Harini.K and Geetha.R.V. This is an open access article distributed under the Creative Commons Attribution License, which permits unrestricted use, distribution, and reproduction in any medium, provided the original work is properly cited.

\section{INTRODUCTION}

Enterococci are gram positive cocci that can occur singly, in pairs, or as short chains. They are facultative anaerobes, possessing the ability to grow in the presence or absence of oxygen.They catabolize a variety of energy sources including carbohydrates, glycerol, lactate, malate, citrate, arginine, agmatine, and many $\alpha$ keto acids(1). It is a member of the mammalian gastrointestinal microbiota but multidrugresistant strains have been considered relevant causes of hospital-acquired and community related infections (2). Enterococci are common bacteria that inhabit the gastrointestinal tract, oral cavity, and vagina of humans and animals (3). Enterococcus faecalis is capable of surviving in a starved environment for a long period of time. It can invade the dentinal tubules and has the property of circumventing the antimicrobial effect of calcium hydroxide. It has several virulence factors such as aggregation substance, surface adhesions, gelatinase, and toxic cytolysin(4).

*Corresponding author: Harini.K

Saveetha Dental College and Hospitals, Chennai, India
Enterococcus faecalis is often isolated from previously treated teeth with persistent diseases (5). Isolates from oral infections differ from clusters of hospital-derived isolates, as they do not present many mobile genetic elements. However, they usually carry virulence factors related to adhesion and biofilm formation, which may account for the colonization of different oral sites (2). The microenvironment of root canals may especially favour the survival of enterococci and the establishment of long-standing local infections. Other conditions, such as the quality of obturation, could conceivably also influence the colonization of $\mathrm{E}$. faecalis and hence microflora in roots, either directly or indirectly (3). E. faecalis can adhere to root canal walls, accumulate, and form communities organized in biofilm, which helps it resist destruction by enabling the bacteria to become 1000 times more resistant to phagocytosis, antibodies, and antimicrobials than non-biofilm-producing organisms (6). The aim of this article is to evaluate the phenotypic characteristics of Enterococcus faecalis isolated from endodontic infections. 


\section{MATERIALS AND METHODS}

A total of 20 samples were collected from patients undergoing endodontic treatment. The samples were inoculated in Mac Conkey agar and brain heart infusion agar. Presumptive identification of Enterococci was done by Gram's stain, Catalase test and Heat tolerance test in which Enterococci are gram positive cocci arranged in pairs. Catalase negative, tolerance the temperature of $60^{\circ} \mathrm{C}$ for $30 \mathrm{mins}$ respectively. On Mac Conkey agar they showed small Lactose fermenting colonies.

\section{Detection of haemolytic activity and gelatinise activity}

Brain-heart infusion agar (Oxoid) supplemented with 5\% sheep blood was used for the detection of haemolytic activity. Pure isolates were cultivated on blood agar plates and plates were incubated at $37^{\circ} \mathrm{C}$ for $24 \mathrm{~h}$. Haemolytic activity was observed as $(\beta)$ haemolysis surrounding bacterial colonies. (Complete haemolysis appeared as clear zones).

Gelatinase assay was carried out by adding an inoculum from a pure culture into tubes containing $12 \%$ gelatin in $0.8 \%$ nutrient broth. Tubes were incubated for $24-72 \mathrm{~h}$ at $37^{\circ} \mathrm{C}$ and then placed in the refrigerator for approximately $30 \mathrm{~min}$. The liquefaction of gelatin was considered as positive result.

\section{RESULTS AND DISCUSSION}

Enterococcus faecalis posses cytolysin or hemolysin as a virulence factor. Conflicting studies suggesting the role of cytolysin as a possible virulence factor. Initial studies reported that approximately $60 \%$ of Enterococcus faecalis isolates derived from fecal specimens from healthy individuals. However recent studies show that the role of cytolysin as a virulence factor is small or negligible (7).

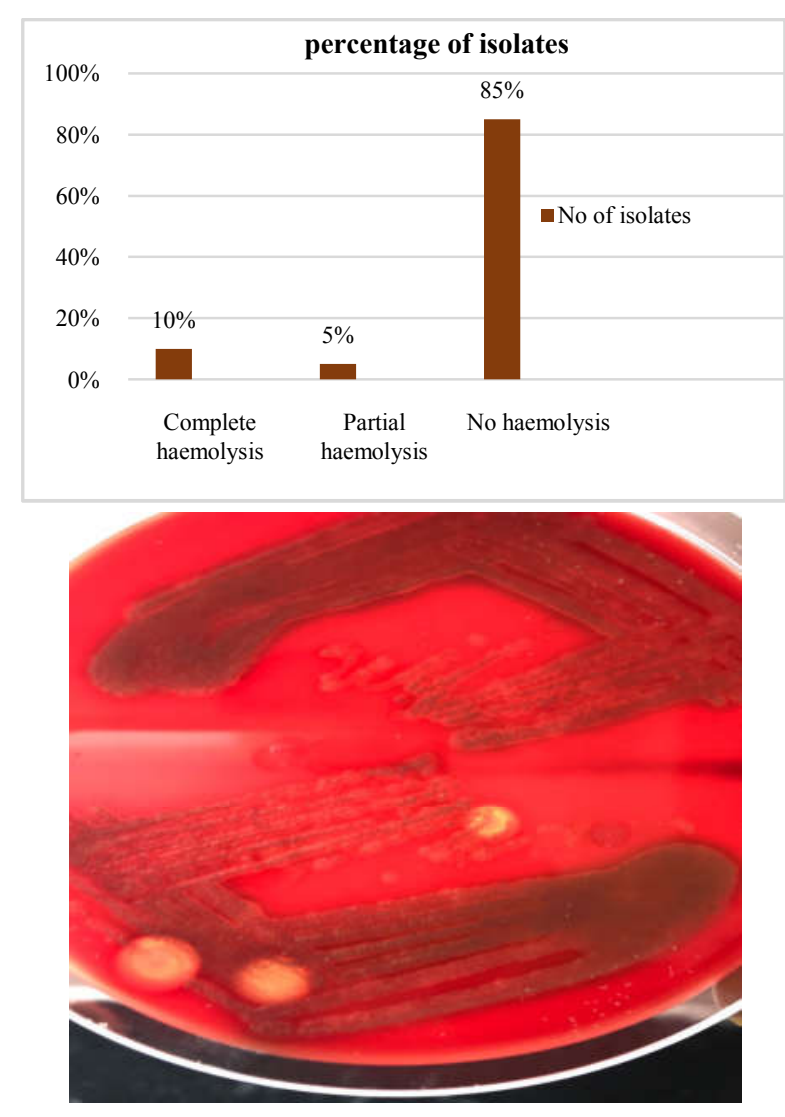

Cytolysin production was screened by cultivation on blood agar, $2(10 \%)$ isolates produced complete $(\beta)$ haemolysis, $1(5 \%)$ isolate produced partial $(\alpha)$ haemolysis and the rest of the isolates did not produce haemolysis on blood agar $(\gamma)$ haemolysis.

\section{Haemolytic activity}

Enterococcus faecalis possess gelatinase which help it bind to gelatin and gelatinase levels were elevated in oral rises, crevicular fluid and whole saliva samples and in gingival biopsy specimens from periodontitis patients compared with healthy subjects. High gelatinase production has also been seen in epidermiologic studies with human clinical isolates (7).

Gelatinase contributes to the bone resorption and degradation of dentin organic matrix, thus playing an important role in the pathogenesis of periapical inflammation.(8)

Gelatinase assay by gelatin hydrolysis test revealed that $4(20 \%)$ of the isolates phenotypically expressed gelatinase gene as they could not liquefy gelatin media.

Gustavo O. zoletti found all 20(100\%) of strains presented the gelE gene (gelatinase). But, 10(50\%) of them did not hydrolyse gelatin. Seven of the 10 gelatinase producing isolates were recovered from root canals with lesions, which suggests a role for its virulence factor in the pathogenesis of post treatment disease (9).

Renata Ximenes Lins et al found that all 20 isolates (100\%) were positive for gelatinase (gelE) (10).

\section{Gelatin liquefaction test}

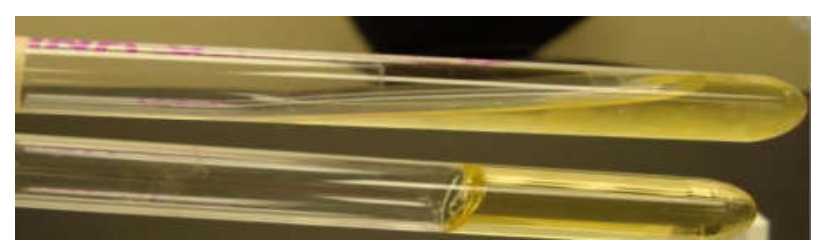

\section{CONCLUSION}

$2(10 \%)$ isolates produced complete $(\beta)$ haemolysis, $1(5 \%)$ isolate produced partial $(\alpha)$ haemolysis and the rest of the isolates did not produce haemolysis on blood agar $(\gamma)$ haemolysis. $4(20 \%)$ of the isolates phenotypically expressed gelatinase gene.We can conclude that there may be a correlation between virulence factors detected and pathogenicity of E. faecalis. Further studies can be done to find the gene for haemolytic and gelatinase activity.

\section{References}

1. Charles H. Stuart, ScottA.Schwaetz, Thomas J. Beeson, Christopher B. Owatz. Enterococcus faecalis: Its role in root canal treatment failure and current concepts in retreatment. JOE vol32, number 2, February 2006.

2. Pinheiro ET, Mayer MPA (2014) Enterococcus faecalis in Oral Infections. J Interdiscipl Med Dent Sci 3: 160. doi: 10.4172/2376-032X.1000160

3. Qian-Qian Wang, Cheng-Fei Zhang, Chun-Hung Chuand Xiao-Fei Zhu. Prevalence of Enterococcus faecalis in saliva and filled root canals of teeth associated with apical periodontitis. International Journal of Oral Science (2012) 4, 19-23. 
4. Bharati D. Deo, Shashikala K. and Kishore G. Bhat. Detection of Enterococcus Faecalis In Symptomatic And Asymptomatic Endodontic Infections By Culture And Pcr Method.World Journal of Pharmaceutical and Medical Research. 2016,2(6), 62-68

5. Thomas R.Dunavant, John D.Regan, Gerald N.Glickman, Eric S.Solomon, Allen L.Honeyman. Comparative evaluation of endodontic irritants against Enterococcus faecalis biofilms. JOE-vol 32, number 6,2006.

6. Rashmirekha Mallick, Sandhyarani Mohanty, Subasish Behera, Priyanka Sarangi, Soumyaranjan Nanda, Sukanta Kumar Satapathy. International Journal of Contemporary Dental and Medical Reviews (2014), Article ID 0111145.

7. Sonia Bhonchal Bhardwaj. Role of Enterococci faecalis in failure of Endodontic treatment. International journal of current microbiology and applied sciences. ISSN: 2319-7706 Volume 2 Number 8 (2013) pp. 272277 http://www.ijcmas.com
8. Rashmirekha Mallick, Sandhyarani Mohanty, Subasish Behera, Priyanka Sarangi,Soumyaranjan Nanda, Sukanta Kumar Satapathy. Enterococcus faecalis: A resistant microbe in endodontics. International Journal of Contemporary Dental and Medical Reviews (2014), Article ID 011114.

9. Gustavo O. Zoletti. Eliezer M. Pereira, Ricardo P. Schuenck, Lucia M. Teixeira, Joseo F. Siqueira Jr., Katia Regina N. dos Santos. Characterization of virulence factors and clonal diversity of Enterococcus faecalis isolates from treated dental root canals. Research in Microbiology 162 (2011) 151e158

10. Renata Ximenes Lins, Aurimar de Oliveira Andrade, Raphael Hirata Junior, Melanie J. Wilson, Michael A.O. Lewis, David W. Williams, Rivail Antonio Sergio Fidel. Antimicrobial resistance and virulence traits of Enterococcus faecalis from primary endodontic infections. Journal of dentistry 4 I(2013) 779 -789.

\section{Please cite this article in press as:}

Harini.K and Geetha.R.V (2017), Phenotypic Characteristics of Enterococcus Faecalis Isolated from Endodontic Infections, International Journal of Current Advanced Research, 6(3), pp. 2721-2723.

http://dx.doi.org/10.24327/ijcar.2017. 2723.0088 